\title{
Experimental and Numerical Investigation of Large Scale Structures in Cavitating Wakes
}

\author{
Roger EA Arndt ${ }^{*}$ and Martin Wosnik ${ }^{\dagger}$ \\ and \\ Qiao Qin ${ }^{\ddagger}$
}

\begin{abstract}
Cavitation is a design consideration for a broad variety of devices handling liquids. In many cases, unstable operation is caused by cavitation-induced flow instabilities. Complex cavitation characteristics are observed in many types of fluid machinery. Examples range from the highpressure fuel pumps in the Space Shuttle Main Engine to a variety of hydroturbines. In addition there is an increasing interest in very high performance marine vehicles that must operate in the cavitating regime. Associated with the deleterious effects of performance breakdown, noise, and vibration, there is a possibility of erosion. The purpose of this research is to investigate the twophase flow structure in the wake of a hydrofoil undergoing unsteady partial cavitation using an integrated experimental/numerical approach. This topic provides both numerical and experimental challenges. A two-dimensional NACA 0015 hydrofoil was selected for study, because of its previous use by several investigators around the world. The simulation methodology is based on a Large Eddy Simulation (LES), using a barotropic phase model to couple the continuity and momentum equations. The complementary experiments were carried out at two different scales in two different water tunnels. Tests at the St. Anthony Falls Laboratory (SAFL) were carried out in a $0.19 \times 0.19 \mathrm{~m}^{2}$ water tunnel and a geometrically scaled up series of tests was carried out in the $0.3 \times 0.3 \mathrm{~m}^{2}$ water tunnel at the Versuchsanstalt für Wasserbau (VAO) in Obernach, Germany. The tests were designed to complement each other and to capitalize on the special features of each facility.
\end{abstract}

\section{Nomenclature}

$\mathrm{c} \quad=$ chord length

$\mathrm{f} \quad=$ frequency

$1=$ cavity length

$\mathrm{p}_{\propto} \quad=$ upstream pressure

$\mathrm{p}_{\mathrm{v}} \quad=$ vapor pressure

$\mathrm{U}_{\infty} \quad=$ upstream velocity

$\alpha \quad=$ angle of attack

$\rho \quad=$ density

$\sigma \quad=$ cavitation number

\section{Introduction}

Cavitation is a design consideration for a broad variety of devices handling liquids. Examples range from the high-pressure fuel pumps in the Space Shuttle Main Engine to a variety of hydroturbines and marine propellers. In addition there is an increasing interest in very high performance marine vehicles that must operate in the cavitating regime. Associated with the deleterious effects of performance breakdown, noise, and vibration, there is a possibility

\footnotetext{
* Professor, St. Anthony Falls Laboratory, University of Minnesota, Minneapolis, MN 55414, Associate Fellow

${ }^{\dagger}$ Research Associate, St. Anthony Falls Laboratory, University of Minnesota, Minneapolis, MN 55414, Member

${ }^{\ddagger}$ Director of Global Engineering, White Drive Products, Hopkinsville, KY 42240
} 
of erosion (Arndt, 1981) [1]. In many cases, unstable operation is caused by cavitation-induced flow instabilities. Cavitation is also known to produce air bubbles due to incondensable gas coming out of solution in low-pressure (supersaturated) regions of the flow. The production of bubbly flows in hydraulic equipment can have insidious effects on the stability of operation and on vibration. There are a variety of references in the literature that describe the interrelation between cavitation performance and dissolved air dating back as far as 50 years ago (Edstrand, 1950) [2]. However, a quantitative understanding of the interrelation between dissolved gas and cavitation phenomena is still beyond our grasp.

A particularly important form of cavitation from a technical point of view is attached cavitation on the suction side of lifting surfaces. At typical angles of attack, this takes the form of a sheet, often terminated at the trailing edge by a highly dynamic form of cloud cavitation. Vortex cavitation is often observed in the cloud, which is caused by vorticity shed into the flow field. These cavitating microstructures are highly energetic and are responsible for significant levels of noise and erosion.

Cavitating flows are challenging to model numerically, since they are turbulent, highly dynamic and highly unstable two-phase, gas-liquid flows. The problem with commonly used potential flow or boundary element approaches is that it is beyond their capability to model the closure region near the trailing edge where the cavity collapses. There currently is no physical model that satisfactorily describes the phenomena associated with cavitation in the closure region. However, the events in this closure region are important, since the dynamics of oscillating partial cavities, and the associated dynamics of lift, are closely coupled to them. One way of working around this lack of a physical model is to use Direct Numerical Simulation (DNS) of the single-phase Navier-Stokes equations, or, if one wants to go to larger, realistic Reynolds numbers, Large Eddy Simulation (LES).

A possible simplification of complex cavitating flows is to assume the gas-liquid flow as a virtual single phase fluid with a sharp density change when pressure drops below some critical value (Kubota et al. 1992, Song et al. 1997) [3,4]. Promising results have been obtained with these simulations, indicating that the approach can capture the main physics of cavitating flows (Song and Qin, 2001, Qin et al. 2003, Arndt et al. 2004, Qin 2004) [5-8]. Largescale three-dimensional cavitating structures can be found in the wake of two-dimensional hydrofoils, as a result of sheet/cloud cavitation on the suction side. Sheet cavities are formed, "pinched off" and shed into the wake of a cavitating hydrofoil as vaporous/gaseous clouds. This type of cavitation produces unsteady lift on most hydrofoils, including the NACA 0015 hydrofoil studied here, but is periodic and therefore offers the potential for control.

The general features of the wake are illustrated in Figure 1, where the large lateral excursion of flow structures in the wake is evident, quite different from the relatively thin viscous wake that would occur at this angle of attack under non-cavitating conditions. The periodic shedding of vortical clouds of bubbles into the wake is associated with lift oscillations, the amplitude of which can exceed $100 \%$ of the steady state lift. The spectral characteristics of cavitation-induced lift oscillations vary considerably over a measured range of $1.0 \leq \sigma / 2 \alpha \leq 8.5$ (Arndt et al. 2000) [9]. $\sigma$ is the cavitation number, defined as

$$
\sigma=\frac{2\left(p_{\infty}-p_{v}\right)}{\rho U_{\infty}^{2}}
$$

and $\alpha$ is the angle of attack. Three different types of oscillatory behavior have been reported in Arndt et al. (2000). Different oscillatory behavior is generally associated with different types of cavitation. This is illustrated in Figure 2 where cavitation behavior over an envelope of angle of attack and cavitation number is depicted. The regions depicted by I and II correspond to sheet/cloud cavitation (the focus of this paper). Note that regions I and II have different spectral characteristics as shown in Figure 3. A bifurcation in the data occurs at $\sigma / 2 \alpha=4$. When $\sigma / 2 \alpha \leq 4$ a strong spectral peak exists at a Strouhal number, $\mathrm{fc} / \mathrm{U}$, ranging from about 0.15 to 0.3 that is independent of cavitation number. Under these conditions, the relative maximum cavity length

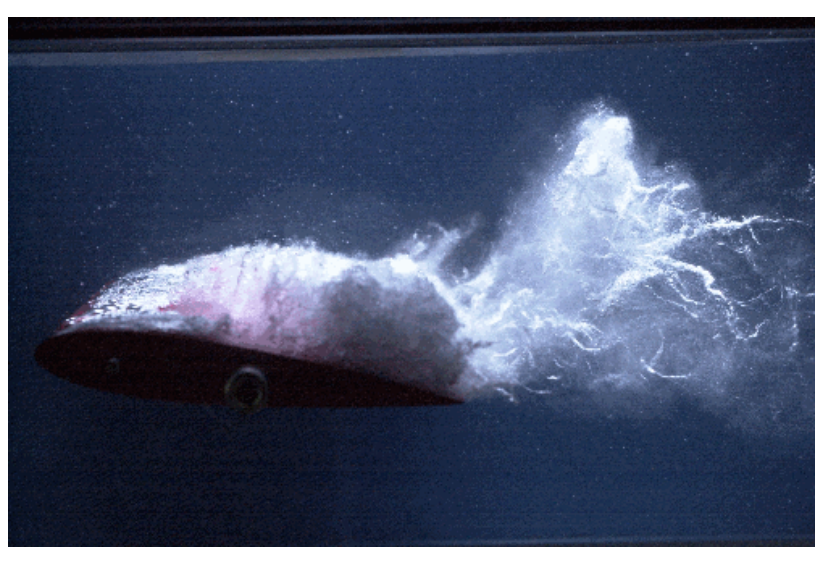

Figure 1. The wake behind a cavitating NACA 0015 hydrofoil at an angle of attack of $8^{\circ}$. 


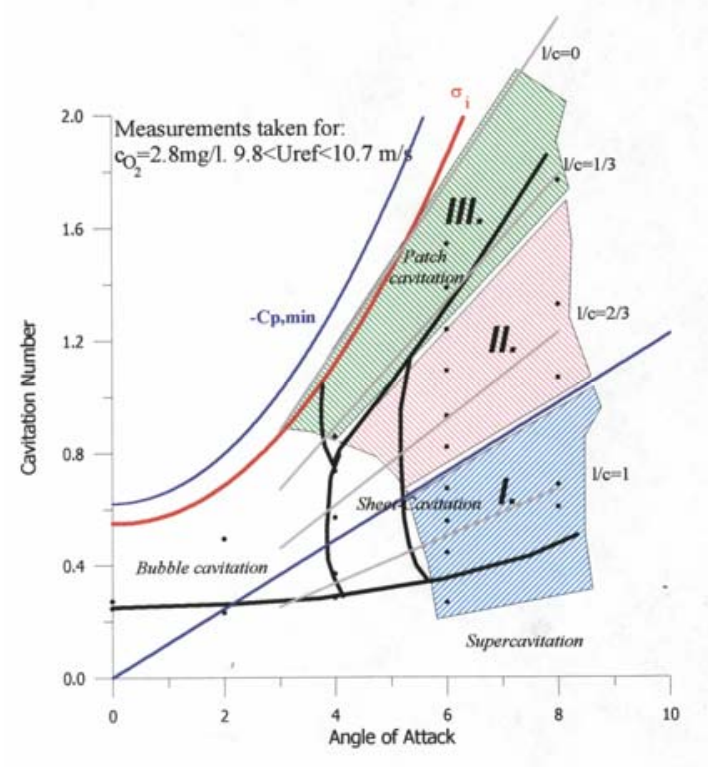

Figure 2. Types of cavitation found on the NACA 0015 hydrofoil.

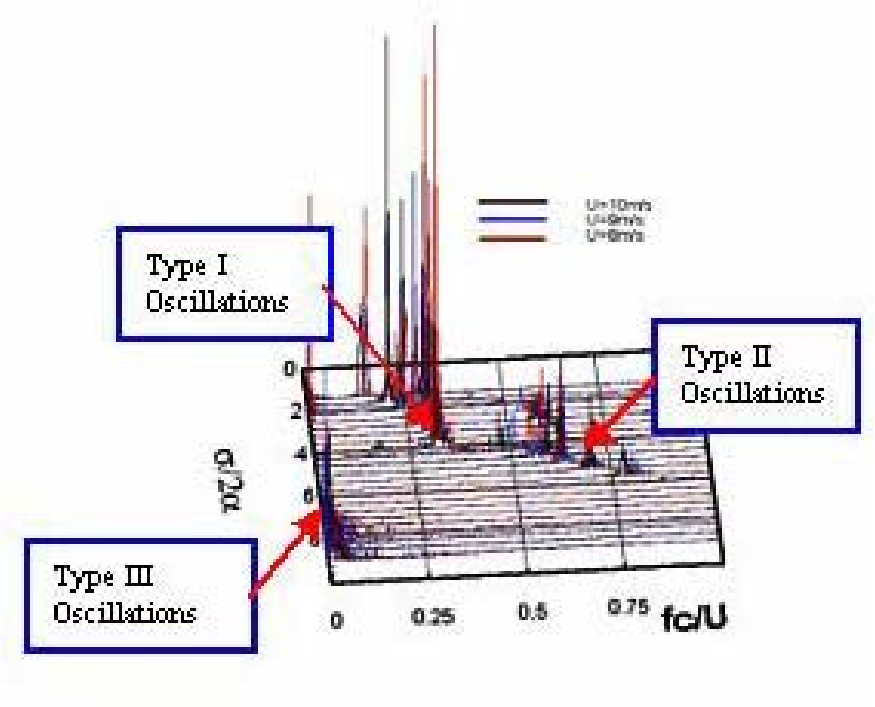

Figure 3. Spectra of measured lift oscillations.

is greater than $0.75(\mathrm{l} / \mathrm{c}>0.75)$. At values of $\sigma / 2 \alpha \geq 4$, a different mode of oscillatory behavior (Regime II) is noted. This corresponds to cavity lengths that are less than $0.75 \mathrm{c}$ and peak frequency scales with cavity length $\mathrm{l}, \mathrm{fl} / \mathrm{U} \approx 0.3$. The importance of this form of sheet cavitation was underscored by Lange et al (1994) [10]. Based on their own observations and a reinterpretation of the results of Kubota et al (1989) [11], they suggest that a re-entrant jet within the sheet is the mechanism for regular breakup of sheet cavitation into large cylindrically shaped bubble clouds. As a re-entrant jet is formed, it moves forward to the leading edge of the cavity, colliding with the cavity interface and pinching off the cavity. The rearward part of the sheet forms a bubble cloud. The concept of a reentrant jet is appealing since a simple model can be used to describe the general features. Using a model that assumes that of rate vorticity introduced at the cavity detachment can be equated to the time rate of change of circulation in the sheet, Arndt et al (1995) [12] found that $\mathrm{fl} / \mathrm{U}=0.25(1+\sigma)^{1 / 2}$, which is in qualitative agreement with the data of de Lange (1996) [13] and Kawanami et al (1998) [14]. This simple concept can also explain the observation that periodic fluctuations in lift occur because of periodic shedding of vortical clouds of cavitation into the wake.

The significance of the bifurcation at $\sigma / 2 \alpha=4$, is discussed by Watanabe et al (1998) [15]. Their results, based on an inviscid analysis of flow over a flat plate, show that the flow becomes unstable when $\mathrm{l} / \mathrm{c}$ is greater

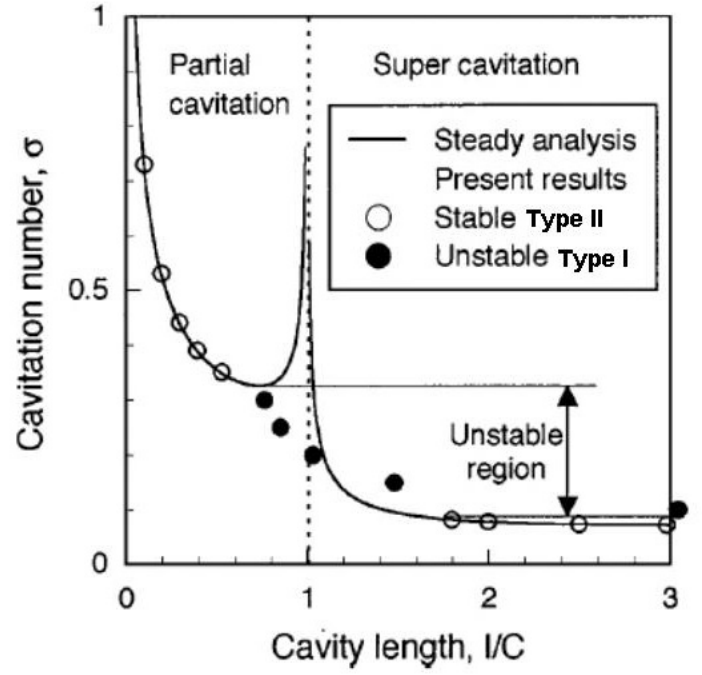

Figure 4. Analysis of cavitating flow over a flat plate (Watanabe et al, 1998).

than 0.75 . They refer to this regime as transitional because of the discontinuity between partial and super cavity solutions as shown in Figure 4. The simulations and experiments presented in the remainder of this paper focus on transitional sheet/cloud cavitation regime (Type I) where $\sigma / 2 \alpha \leq 4$ and both bubbly flow shock wave and re-entrant jet phenomena can occur (as opposed to reentrant jet physics dominating for values of $\sigma / 2 \alpha$ greater than 4 ). This is a more complex form of sheet cloud cavitation that defies simple analysis. Further insight into this regime required an integrated numerical/experimental approach. 


\section{Wake Physics}

Arndt et al. (2000) [9] conducted measurements in the wake of a NACA 0015 hydrofoil under both cavitating and non-cavitating conditions using an LDV (Laser Doppler Velocimetry) system. It was found that self-similarity of the wake shape existed quite close to the trailing edge, for both cavitating and non-cavitating flows. Mean velocity profiles for cavitating and non-cavitating conditions collapsed reasonably well using the classic similitude of a turbulent wake. It was also found that the averaging process obscured much of the important physics in the process. The non-cavitating LDV signal resembled a typical turbulence signature, while the cavitating signal is skewed towards lower velocity. The strong negative fluctuations in velocity were due to the imprint left by the periodic passage of vortices from cavitation processes that extended much further from the wake centerline than from a typical non-cavitating viscous wake.

In order to improve on the observations of Kjeldsen et al, (2000) [16] phase-locked, time-resolved photography triggered off piezoelectric pressure sensors (essentially providing instantaneous pressure iso-surfaces of a cavitating flow) qualitatively correlates well with the LES in the wake of the hydrofoil. For quantitative measurement in cavitating flows with hopes of finding flow structures one must resort to Particle Image Velocimetry (PIV), since a technique like Laser Doppler Velocimetry (LDV) only provides quasi-point measurement of mean flow and statistics. Because standard, low repetition-rate PIV systems are limited to instantaneous "snapshots" and statistical information, they can shine little light on the evolution of flow structures (which are being shed at inverse time scales of tens of, and sometimes several hundred Hz). Time-Resolved Particle Image Velocimetry (TR-PIV), on the other hand, can provide whole-field, time-resolved, quantitative experimental velocity. The typical way to employ PIV in cavitating flows is to use fluorescent seeding particles, and then optically (usually long-pass) filter the scattered light so that only the fluorescent light from the particles, but not the direct reflections from the bubbles are recorded on the camera. The region where cavitation occurs is then identified by some masking technique, and excluded from cross-correlation processing. The result is the liquid velocity field with identified regions of cavitation (e.g., Laberteaux and Ceccio, 2001a,b) $[17,18]$. On the other hand, if one is after velocity information inside cavitation clouds, the vapor/gas bubbles can be used as "particles" (Arndt et al, 2002, 2005) [19, 20]. Only the gas phase velocity is then being measured. This had been applied successfully to bubbly wakes resulting from airventilated cavities by Wosnik et al. (2004) [21], but is somewhat more problematic in natural, vaporous/gaseous cavitation since "seeding particles" can simply disappear between frames due to condensation.

Our recent numerical simulations have uncovered new details about the cavitating wake, which were not observed experimentally. The reason for this is that experimental data were mostly time averaged. Qin (2004) [8] investigated the unsteady and vortical structures behind a cavitating hydrofoil using a virtual single-phase cavitating model with a barotropic flow assumption, initially without consideration of the dissolved gas effect. Five types of unsteady vortex shedding mechanisms are identified. A pair of primary vortices consisting of a cloud cavity and an induced positive vortex is shed periodically into the wake region. Between two pairs of primary vortices, there are secondary pairs of vortices shed into to the wake in a less periodical manner. There are also strings of positive vortices formed between primary and secondary pairs of vortices. These vortices have the appearance of a semiKarman Vortex Street. The lift coefficient of the cavitating hydrofoil oscillates in a manner that is highly correlated with the shedding of the described vortical structures in the wake.

A detailed near field study by Qin et al. (2003) [6] also shows that the maximum lift occurs at the time of maximum cavity length. Minimum lift (lift can be negative) occurs when the cloud cavity is about to leave the trailing edge. From the time when the sheet cavity is broken off by the reentrant jet, cavitation starts anew from the leading edge towards the trailing edge. Lift decreases with time during this period. As the cloud cavity leaves the hydrofoil and the boundary layer is being restored the lift increases and a new sheet cavity grows in size.

Although the averaged numerical data agree very well with the experimental data in the simulation, it was found that the numerical simulations were only able to fit the data close to the trailing edge, but deviated from the measurements further downstream. It is believed that this is an effect of dissolved gas that has come out of solution. The original numerical model considers only water vapor that will condense quickly as the pressure rises downstream, while gas bubbles will persist in the wake for a much longer period of time after they are out of solution. A revised numerical model that takes into account of the incondensable gas has shown that including the effect of incondensable gas results in excellent agreement with the experimental data.

It was also natural to conclude that the gas content in the wake will show a cyclic behavior (Kjeldsen et al, 2000) [16]. The observed cyclical increase in the data rate of LDV measurements gives additional information regarding cavitation dynamics. In comparing the LDV frequency domain fingerprint with corresponding lift, similarities were found that identified a strong correlation between lift oscillations and vortex shedding. 


\section{Numerical and Experimental Techniques}

\section{Description of LES approach}

The Large Eddy Simulation (LES) is based on a virtual single fluid model with a barotropic flow assumption. Above a certain critical pressure the model is considered "weakly compressible", below the critical pressure "fully compressible" with a sharp density change. Motivated by an initial discrepancy with experimental mean wake data (LDV), the formulation was expanded to include dissolved incondensable gas coming out of solution below the critical pressure.

The computational domain is shown in Figure 5. The Reynolds number for the LES based on free stream velocity and chord length was $10^{6}$. The LES code is a three-dimensional code, but due to computational constraints only 5 grid points (including two phantom points) were used in the spanwise direction. The mesh structure of the computational domain, c.f. Figure 6, is heavily clustered near the solid surface of the hydrofoil, since the flow near the surface and in the near-wake are of main interest. A finite volume method with a $2^{\text {nd }}$ order accurate MacCormack predictor-corrector scheme was used. For detail of the numerical simulation see Qin (2004) [8].

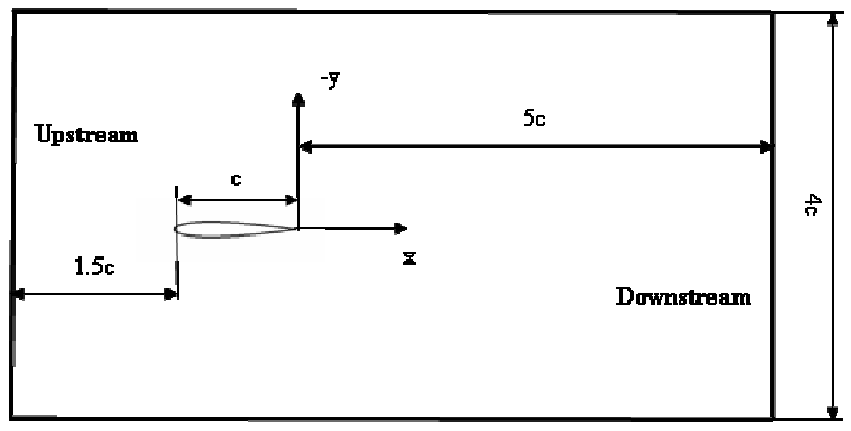

Figure 5: Computational domain for Large Eddy Simulation (LES) of NACA 0015.

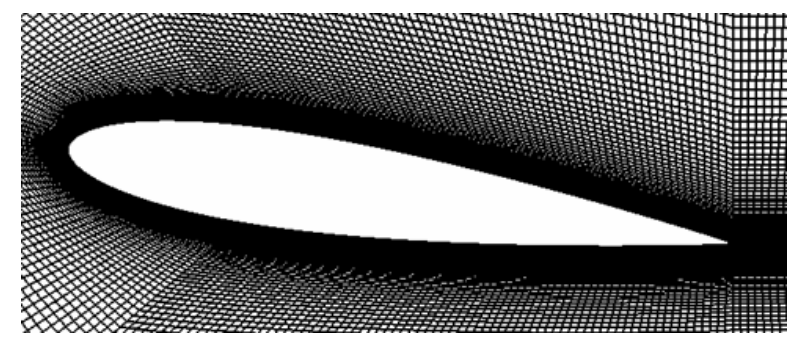

Figure 6: Computational mesh around hydrofoil at angle of attack.

\section{Description of time-resolved PIV setup}

The experiments were carried out in the high-speed water tunnel at St. Anthony Falls Laboratory (SAFL), a recirculating, closed-jet facility with absolute pressure regulation capable of velocities of more than $20 \mathrm{~m} / \mathrm{s}$. The test section measures $0.19 \mathrm{~m}(\mathrm{~W}) \times 0.19 \mathrm{~m}(\mathrm{H}) \times 1 \mathrm{~m}(\mathrm{~L})$, and allows optical access from three sides. An NACA 0015 hydrofoil with $\mathrm{c}=81 \mathrm{~mm}$ chord was mounted horizontally in the test section at an angle of attack of -8 degrees. The hydrofoil is instrumented with a series of piezoelectric pressure transducers and static pressure taps.

The high-speed PIV system was provided by TSI Inc. and consisted of a NewWave Research Pegasus dual cavity Nd:YLF laser $(\lambda=527 \mathrm{~nm})$, a Photron Ultima APX CMOS camera (2000 fps at full-frame 1024x1024 pixel) and timing electronics with 1 ns temporal resolution. Data were acquired and processed with Insight 3G software (=3rd generation, or Insight 7). Image pairs were initially recorded at 1000 and $2000 \mathrm{~Hz}$, where the YLF laser has its 
maximum energy of around $10 \mathrm{~mJ} / \mathrm{pulse}$. This corresponds to camera frame rates of $2000 \mathrm{fps}$ at $1024 \times 1024$ pixel and $4000 \mathrm{fps}$ at $1024 \times 512$ pixel, respectively. However, for the actual data acquisition only the $2000 \mathrm{~Hz}$ (4000 fps) recording rate at 1024 x 512pixel was used. The field of view was $74.7 \times 37.4 \mathrm{~mm}$, leading to a resolution of $73 \mu \mathrm{m} /$ pixel, or a system spatial resolution of $2.3 \mathrm{~mm}$ with a $32 \times 32$ pixel interrogation spot size.

With a free-stream velocity (in front of the hydrofoil) of $7.1 \mathrm{~m} / \mathrm{s}$ and a chord length of $81 \mathrm{~mm}$ the Reynolds number for the experiment was $0.6 \times 10^{6}$. The cavitation number $\sigma$ was varied from 0.63 to 1.66 (corresponding to $\sigma / 2 \alpha=2.26 \ldots 5.94)$ by changing absolute pressure. Reported here is only one case of sheet/cloud cavitation with $\sigma=1.1$ and $\sigma / 2 \alpha$ of just below 4. PIV image pairs were acquired at $2000 \mathrm{~Hz}$, corresponding to mean flow displacement of $4.7 \%$ of the field of view between captures. With a Strouhal number of around 0.25, 200 PIV image pair captures at $2000 \mathrm{~Hz}$ were sufficient to record 2 full cloud cavitation shedding cycles (total recording time 0.1s). A schematic of the arrangement of the hydrofoil, light sheet and field of view in the water tunnel is shown in Figure 7.

\section{Practical issues of PIV using bubbles/vapor as seeding particles}

Issues of practical importance for PIV in cavitating flows are, e.g., that the bubble concentration must be suitable (high enough) for cross-correlation, and that the laser light sheet can be attenuated or blocked by bubbles, and the choice of PIV processing parameters. When computing velocity vector fields by cross-correlating air bubble images, Fontecha [22] found the Hart algorithm to be more accurate and produce a larger number of valid vectors than the standard FFT algorithm. The Hart correlation method [23] identifies particles (bubbles) based on image intensity gradients, not just absolute intensity. It is thus well suited for varying intensity, relatively poor-quality PIV images of cavitation bubbles. PIV images that are slightly overexposed are often better suited for intensity gradient compression, since the saturated particle image centers have low gradients and will be discarded. The compression rate must be chosen carefully to not lose correlation information. For further details and a parametric study of PIV processing parameters in a bubbly ventilated wake including associated errors see [22].

A special challenge in vaporous cavitation is that the time scale of the phase change from gas to liquid when pressure rises above vapor pressure can be smaller than the pulse separation time, i.e., individual bubbles can simply disappear between the firing of laser 1 and 2 during a capture. Another source of losing correlation is the large outof-plane motion in the cavitating wake.

\section{Orientation of mean velocity deficit in non-cavitating and cavitating wake}

A non-cavitating hydrofoil at an angle of attack will create a "downwash" (here upward due to negative angle of attack), illustrated by the direction of the velocity deficit in the velocity contour plot in Figure 8. Behind cavitating hydrofoils, on the other hand, the wake broadens considerably due to cavitation events, creating a velocity deficit directed in the opposite direction, or an "upwash". This is illustrated in Figure 9. Large cross-stream velocities perpendicular to the mean flow can be seen. To facilitate recording of both cases, most of the PIV field of view was located below the trailing edge of the hydrofoil, as shown in Figure 7. Note that only 200 "raw" velocity fields (unfiltered, unsmoothed) were averaged for Figures 8 and 9. Mean velocity deficit profiles from the same two data sets are plotted at selected $x / c$ positions in Figures 10 and 11 using equilibrium similarity variables. The significant wake broadening and change of orientation are also observed. Note that there is significant scatter in the cavitating case , since only 200 vector fields were averaged. 


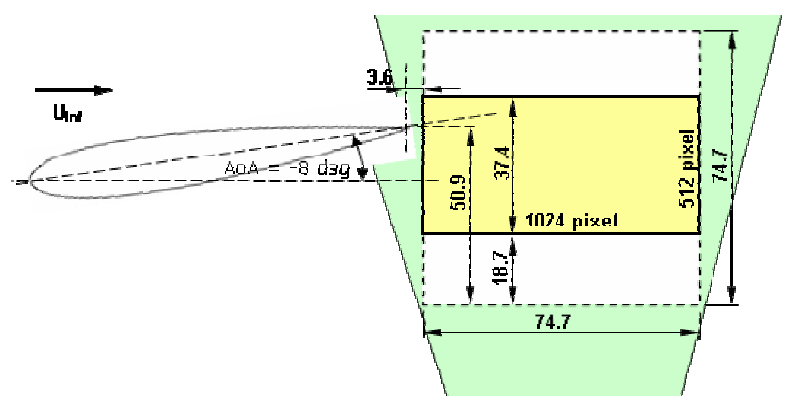

Figure 7. Configuration for time-resolved PIV measurements in the wake of a cavitating hydrofoil. Field of View (yellow) measures 74.7 x 37.4mm (1024 x 512 pixel), and is illuminated by Nd:YLF laser. The instrumented NACA 0015 foil has a chord length $c$ of $81 \mathrm{~mm}$, the angle of attack is -8 degrees.

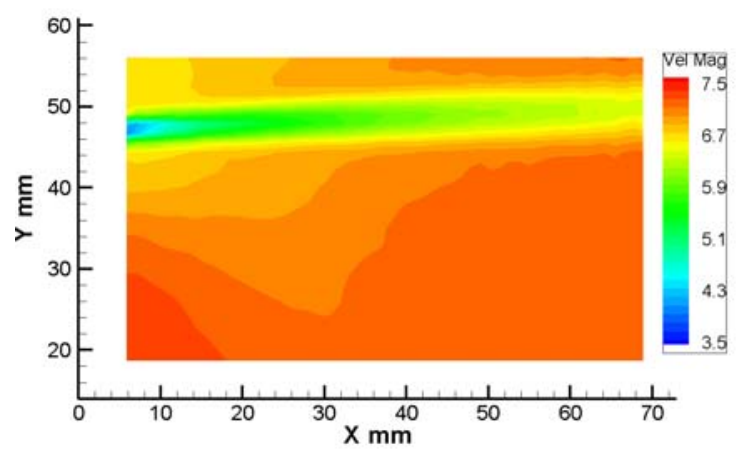

Figure 8. General direction of mean velocity deficit in non-cavitating liquid wake, $\sigma=11.5$ (total recording time was $0.1 \mathrm{~s}$ at $2000 \mathrm{~Hz}, 200$ image pairs, unfiltered/unsmoothed data, velocity in $\mathrm{m} / \mathrm{s}$ ).

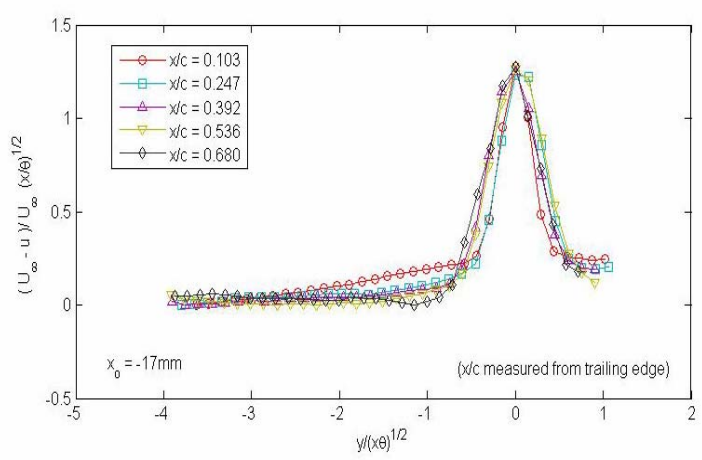

Figure 10. Mean velocity deficit profiles in equilibrium similarity variables (non-cavitating liquid wake, $\sigma=11.5$, same data as in figure 8).

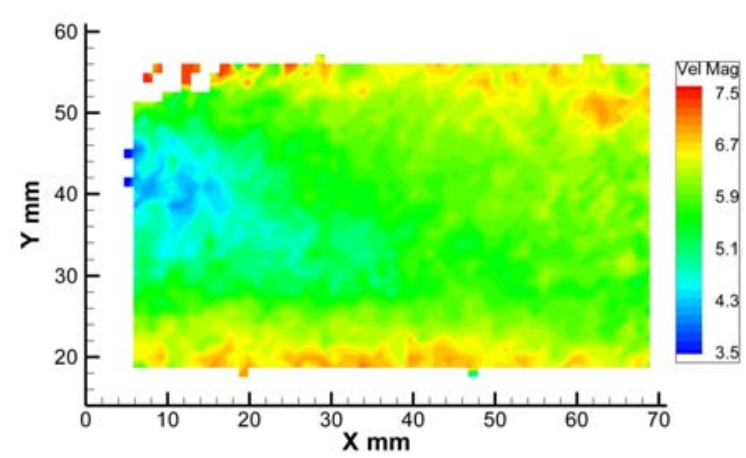

Figure 9. General direction of mean velocity deficit in cavitating wake, $\sigma=1.1$ (total recording time was $0.1 \mathrm{~s}$ at $2000 \mathrm{~Hz}, 200$ image pairs, unfiltered/unsmoothed data, velocity in $\mathrm{m} / \mathrm{s}$ ).

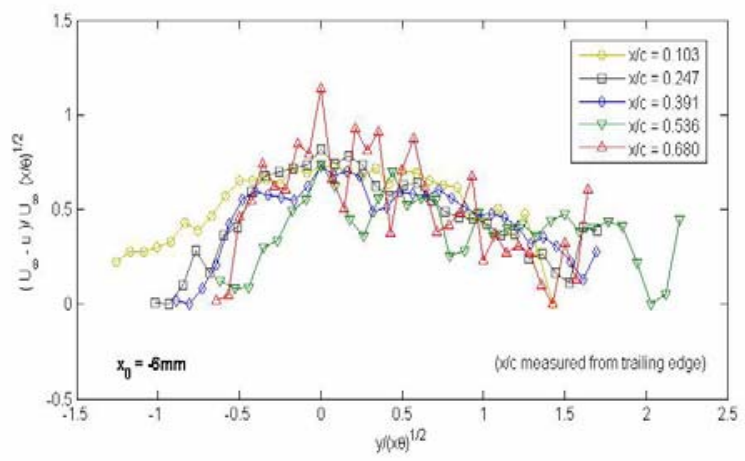

Figure 11. Mean velocity deficit profiles in equilibrium similarity variables (cavitating wake, $\sigma=1.1$, same data as in figure 10). 


\section{Comparison Of Results}

\section{Physical Insight gained from LES - Vortex shedding mechanism}

The LES identified five types of discrete, substantially periodic vortex shedding mechanisms. These mechanisms are illustrated in Figures 12a-d. In particular, cloud cavitation (having a clockwise vortical structure when flow is from left to right) is shed from the suction side of the hydrofoil. This induces a counterclockwise vortex from the pressure side, forming a vortex pair that dominates the wake structure.

The instantaneous velocity field is used to investigate the unsteady vortical structures shed from a cavitating hydrofoil and their evolution in the wake. To facilitate better analysis, the upstream-specified streamwise velocity was subtracted from the instantaneous velocity field of the whole computational domain. Figure 12a illustrates an instance of a large negative (clockwise rotation when flow is from left to right) vortex structure containing a large amount of bubbles being shed into the wake near the trailing edge of the suction side (type A vortex) and a positive (counterclockwise rotation) vortex from the pressure side (type B vortex) that is being induced by the type A vortex. Within a short period of time, a vortex pair containing type A and type B vortices is well formed in the wake immediately downstream of the trailing edge. Since a type A vortex is typically located downstream of a type B vortex, the induced velocities tend to move the vortex pair slightly upward in the cross streamwise direction. As a result, the vortex pair moves diagonally at a speed roughly equal to the free-stream speed (Figure 12b), which results in a slightly slower convection speed of this vortex pair in the streamwise direction. Another observation is that the type A vortex is stronger than the type B vortex so that the relative position of the pair tends to become more parallel to the free stream as they move downstream, which is consistent with the wider spread of a cavitating wake than a non-cavitating wake (Arndt et al, 2000). There is also a secondary negative vortex (type $C$ vortex) formed above the suction side near the trailing edge as is also shown in Figure 12b. This type $C$ vortex is much weaker in strength than the type A vortex, but it also induces a corresponding secondary positive vortex (type $\mathrm{D}$ vortex) from the pressure side and eventually forms a secondary and weaker vortex pair in the wake (Figure 12c). A detailed analysis of the re-entrant jet can explain the secondary negative vortices. When the reentrant jet along the suction side of the hydrofoil starts to impinge on the developed sheet cavity, it usually cannot shear off the whole sheet cavity; instead the sheet cavity is broken by the reentrant jet somewhere close to the leading edge. The broken sheet cavity forms the primary negative vortex. An instantaneous vorticity animation shows that the reentrant jet plays a key

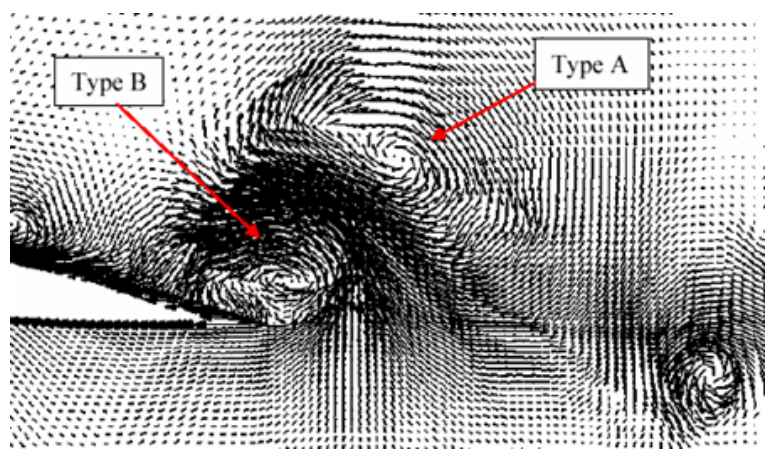

Figure 12a. A primary vortex pair (type A and B) being shed at the trailing edge of a NACA 0015 hydrofoil (note that here the angle of attack is +8 degrees).

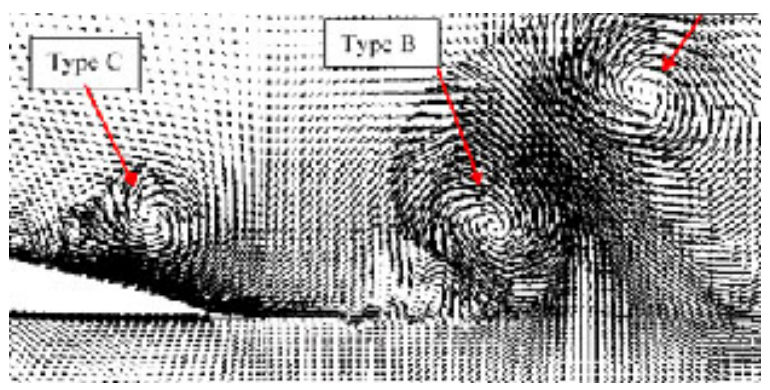

Figure 12b. The primary vortex pair (type A, B) moves into the wake at approximately a 45 degree angle, with the ejection velocity between the vortices A and B pointed at 135 degrees.

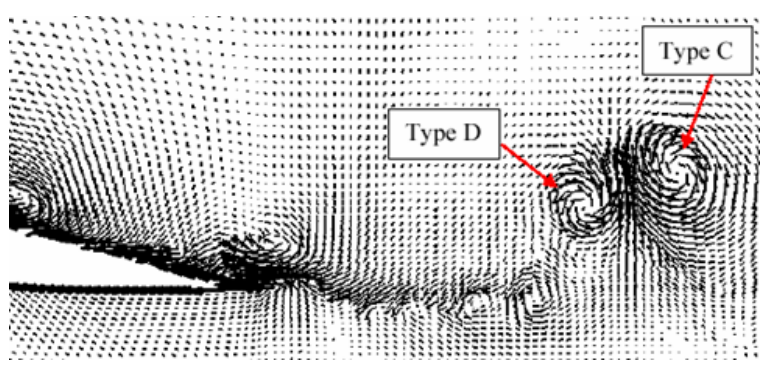

Figure 12c. Secondary vortex pair (type $C$ and $D$ ) moving into the wake.

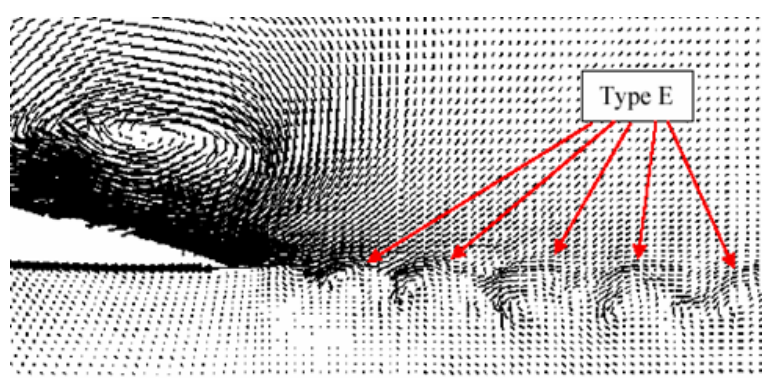

Figure 12d. A period of relative quiescence, or "semi-Karman vortex street" (type E). 
role in the formation of primary and developing in the upstream direction and eventually shears off the rest of the sheet cavity, which is the source of a secondary negative vortex. The primary vortex pair, type A and type B, are periodically shed into the wake while the formation of the secondary vortex pair, type $C$ and type $D$, are less regular. There is typically one secondary vortex pair between two primary vortex pairs, but occasionally two secondary vortex pairs are observed. Between the primary and secondary shedding vortices, there is a certain time period in which only positive vortices (named as type E vortices) are shed from the pressure side of the hydrofoil, shown in Figure 12d.

When the dissolved incondensable gas coming out of solution is superimposed on the velocity vector fields of the primary vortex pair (type A and B), it can be seen that the cavitation cloud being shed appears to coincide with the shedding of the primary vortex pair. This is shown in Figure 8. This can be compared with experiment (phaselocked strobe photography with variable time-delay triggered off pressure peaks on an instrumented hydrofoil) in Figure 13. Note that the image with the numerical result has now been flipped along a horizontal axis to match the angle of attack of -8 degrees of the experiments.

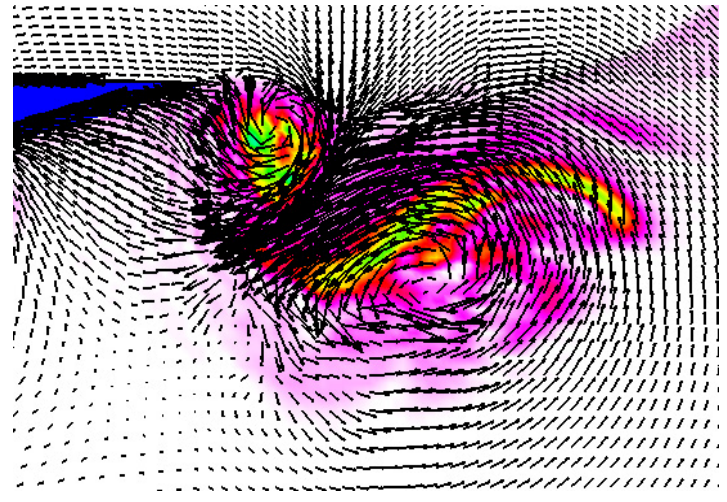

Figure 13. LES of cavitating NACA 0015 hydrofoil, A.o.A. $=-8$ degrees, $. \sigma=1.1$. Type A and $B$ vortices can be seen, the void fraction of incondensable gas is overlaid in color. The cavitation cloud being shed appears to coincide with the shedding of the primary vortex pair.

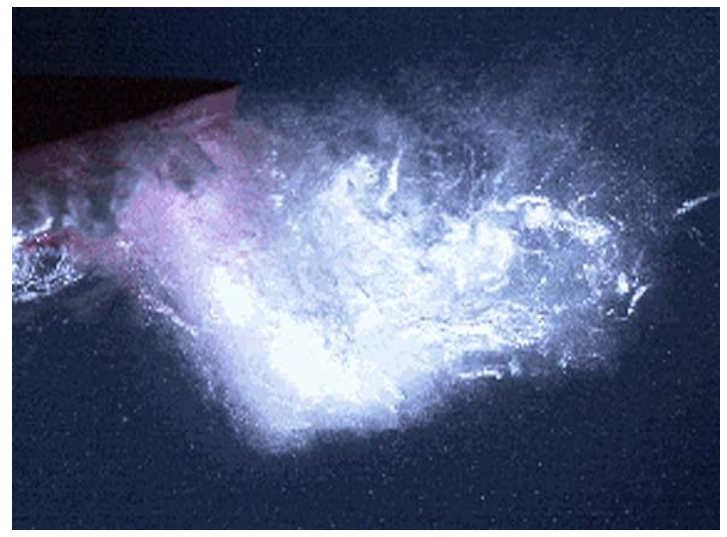

Figure 14. Phase-locked photograph of cloud cavitation.

\section{The three-dimensionality of the cavitating wake of a two-dimensional hydrofoil}

The fact that wakes of cavitating two-dimensional hydrofoils are intrinsically three-dimensional is illustrated in the time-resolved (strobe) photographs in Figures 15 and 16, taken at comparable times during a cavitation cycle. While the side view in both Figures 15 and 16 appears similar at first glance, the simultaneous imaging of the suction side (bottom, plan view) of the hydrofoil clearly shows they are not. The three-dimensionality of the experiment was further documented with high-speed video, also imaging profile and span views simultaneously. This makes comparison of experimental data with the numerical simulation more difficult, mainly since the LES does not provide a sufficient number of grid points in the spanwise direction to allow the largescale structures in the wake to become fully threedimensional.

Initially it was attempted to record phase-locked PIV image captures with a high-resolution, low-repetition PIV system in the wake of the instrumented, cavitating NACA 0015 foil in the following way: Since the lasers did not produce constant power levels for each pulse when externally triggered, both the signal from a pressure transducer on the suction side of the cavitating hydrofoil and the laser 1 Q-switch TTL signal were recorded via a standard data acquisition board. The signal of the pressure transducer was then smoothed and partitioned into "bins". The PIV image pairs were sorted into bins according to when during a cycle they were recorded. A large total number of PIV captures were recorded, since many images per bin were thought to be desirable to build up statistics. However, due to the threedimensionality of the cavitating hydrofoil wake documented in Figures 15 and 16, each bin contained quite varying images, and any sought after vortex structure tended to be "smeared out" in the averaging process. 

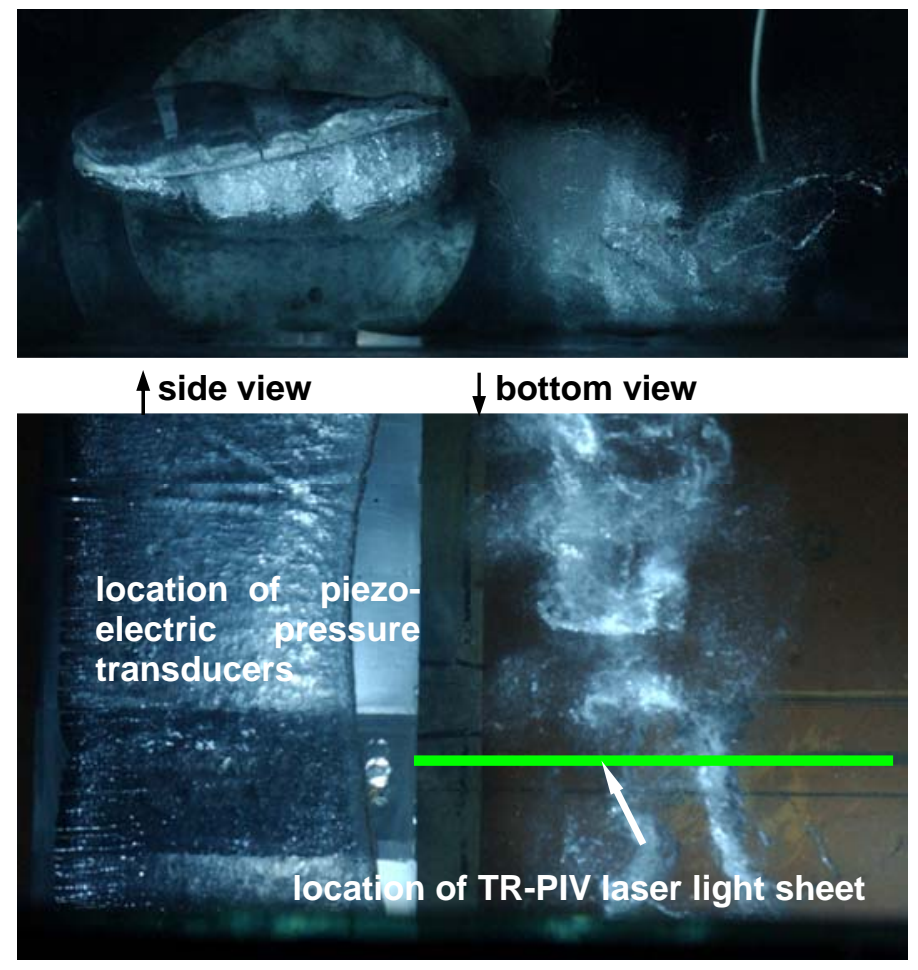

Figure 15. Simultaneous imaging of side and bottom view of sheet/cloud cavitation on a NACA 0015 hydrofoil, showing an example of a relatively uniform cavity development and cloud shedding.

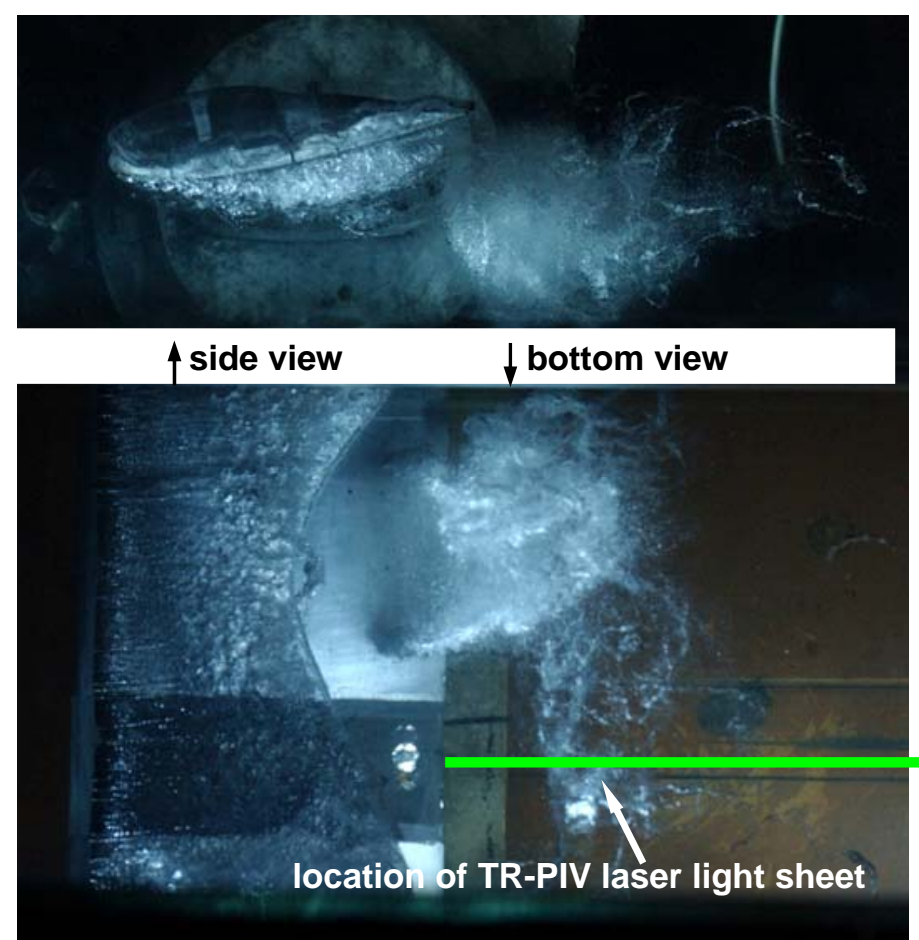

Figure 16. Simultaneous imaging of side and bottom view of sheet/cloud cavitation on a NACA 0015 hydrofoil, illustrating the three-dimensionality of cavity development and cavitation cloud shedding on 2D-hydrofoils

In principle, a hydrofoil could be designed so that the cavitation cloud is shed (mostly) at a predictable spanwise location. This can be accomplished, e.g., by spanwise variation of the angle of attack (thereby creating a three- 
dimensional hydrofoil) which forces the cavitation cloud to be shed predominantly at the location of maximum angle of attack. However, the point of this study is to look at the structures in the wake of a cavitating twodimensional hydrofoil. Time-resolved PIV therefore seemed like the ideal technique to record as well as quantitatively measure the evolution of these structures.

\section{Comparison of LES and TR-PIV}

A typical PIV image acquired after a cavitation cloud has been shed into the hydrofoil wake is shown in Figure 17. In order to only process areas and retain vectors associated with a cavitation event (bubbles), a simple but effective adaptive masking algorithm is applied based on intensity thresholding (sliding filter, matched to PIV interrogation spot size). The result for the image of Figure 17 is shown in Figure 18 (with a masking algorithm normalized intensity threshold of 1.0). Cross-correlation processing was then applied to the unmasked image pair, and the vector matrix was multiplied by the adaptive mask, eliminating interpolated and smoothed vectors in regions without sufficient particles/bubbles. The resulting velocity vectors and vorticity magnitude contours for the mask of Figure 18 are shown in Figure 19.

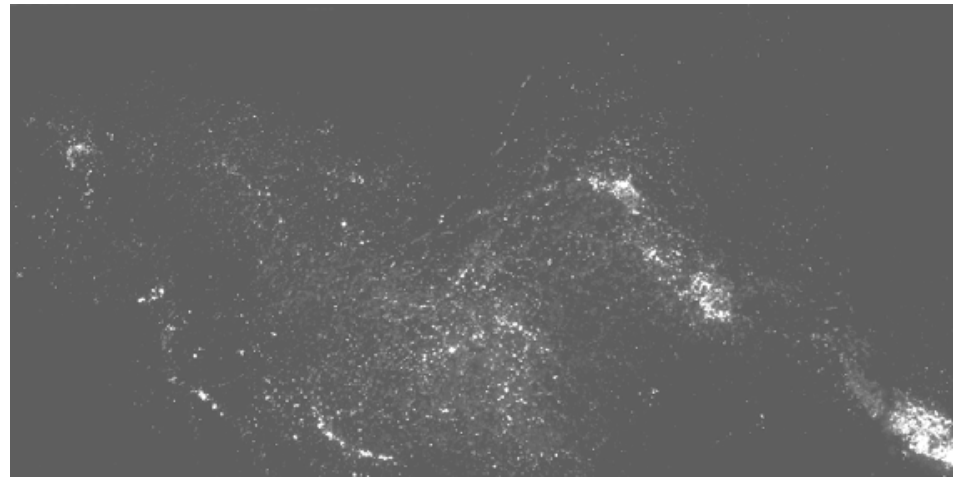

Figure 17. PIV image acquired in cavitating wake (image from laser 1, 1024x512 pixel, c.f. Figure 7). Cavitation number $\sigma=1$.1. Image was brightened for presentation.

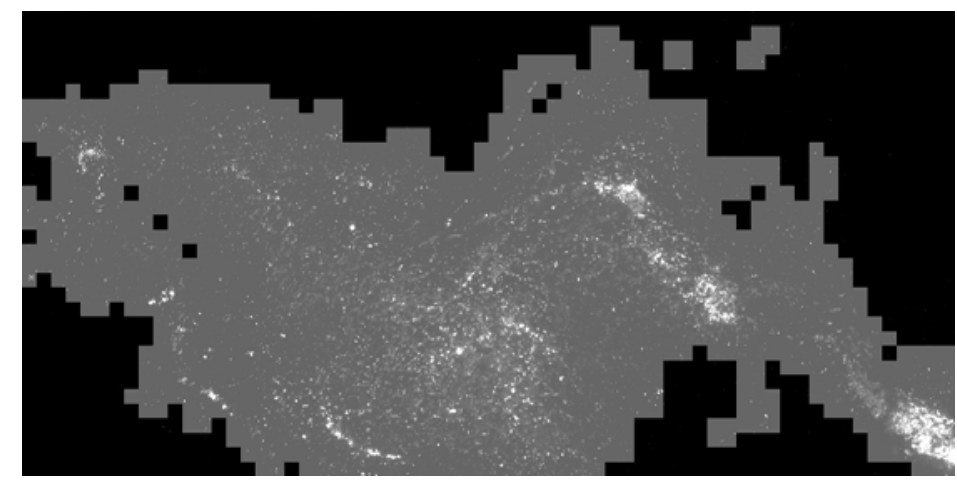

Figure 18. Adaptive processing mask obtained from thresholding 32x32 pixel areas based on the sum of the grayscale intensity values. Overlaid in "active" areas of the mask is the original raw PIV image. 


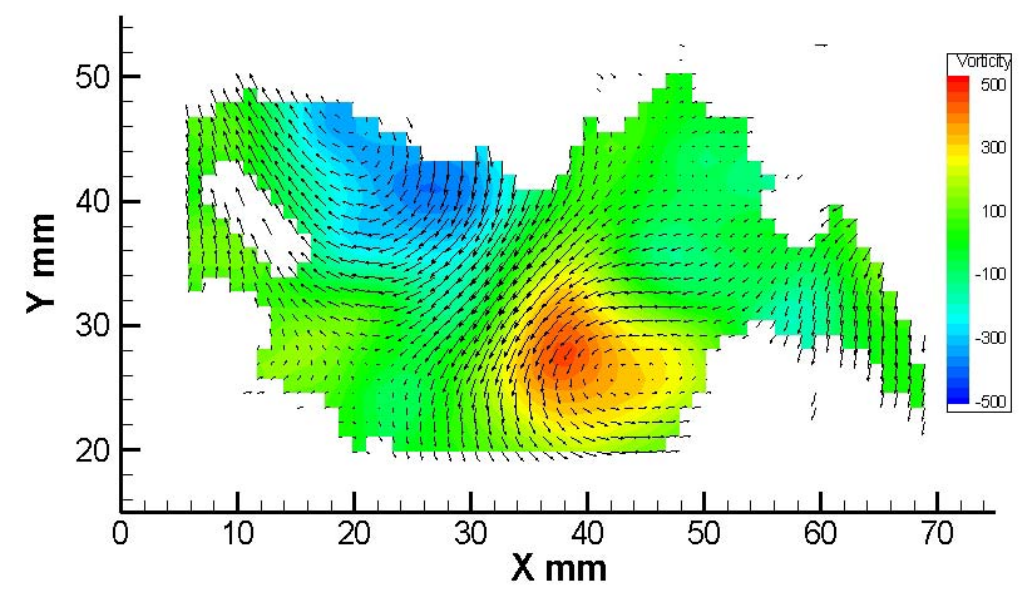

Figure 19. Velocity vectors and vorticity contours in a cavitating hydrofoil. Velocity vectors and contours in a cavitating hydrofoil wake, cavitation number $\sigma=1.1$, from cross-correlation of bubbles. Missing vectors were interpolated, data was smoothed with a Gaussian filter, then vector field matrix was multiplied with mask of Figure 18 (mean velocity subtracted to better visualize vortical motion).

Finally, velocity vectors and vorticity contours are overlaid on the mask and the original image in Figure 20 . The primary vortex pair A-B identified by the LES can clearly be seen in Figures 19 and 20. Figure 20 illustrates how the vortex structure found through PIV image cross-correlation corresponds to the vortical structure observed in the original image or high speed video, and compares well to the LES vector field with incondensable gas overlaid presented in Figure 13. In the PIV data, a cross-stream ejection velocity at an angle of -135 degrees is also observed. The vertical component of this "ejection velocity" can be as large as half the free stream velocity, explaining the considerable broadening of the mean velocity deficit (in this particular capture the maximum V-component is about 2/7 of the free stream velocity). Vortex type A (red, CCW) is slightly stronger than vortex type B (blue, CW). The downstream part of vortex type B (blue) is excluded by the adaptive masking process since it contains bubble-free liquid from the pressure side of the hydrofoil, entrained by vortex type B. On the other hand there are bubbly regions without strong vorticity downstream of the primary vortex pair A-B. These bubbles most likely originated from the same cloud cavitation shedding event, but have no significant out-of-recording-plane vorticity component $\omega_{z}$.

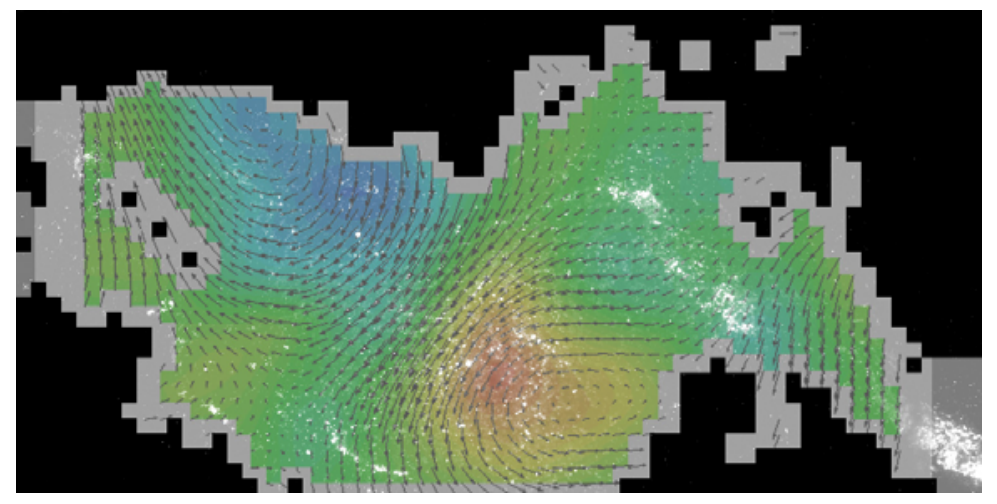

Figure 20. Velocity vectors and vorticity contours overlaid on original image and adaptive mask.

The non-dimensional time step of the LES was 0.0001, and movies of the vortex structure evolution were made with 840 time steps between frames, or a non-dimensional time between frames of 0.084 . At a recording rate of 
$2000 \mathrm{~Hz}$, the non-dimensional time between TR-PIV vector fields is 0.043 . Movies from the simulation can now be compared with movies from the time-resolved PIV data using every second frame. While showing similar vortex structures, the vortex shedding pattern as recorded with TR-PIV is not nearly as regular as predicted by the LES, due the highly three-dimensional nature of the experiment as seen in Figure 16.

\section{Summary and Outlook}

Results from a Large Eddy Simulation (LES) and time-resolved Particle Image Velocimetry (TR-PIV) in the wake of a cavitating NACA0015 hydrofoil were compared. The Large Eddy Simulation (LES) approach for cavitating flow, based on a virtual single-phase, fully compressible cavitation model which includes the effects of incondensable gas, has given detailed physical insight into the complex dynamical features of highly unsteady cavitating flows of two-dimensional hydrofoils. The TR-PIV results confirm the existence of a primary vortex pair (type A-B). In addition to TR-PIV, the cavitation cloud shedding was also documented with phase-locked, timeresolved photography and high speed volume-illuminated video, both with simultaneous imaging of side and plan views of the foil. All three experimental techniques confirm the need for fully three-dimensional simulations to properly describe the unsteady, three-dimensional cavitation cloud shedding mechanism.

Time-resolved PIV holds the promise of providing us with whole-field, high-resolution, time-resolved, quantitative experimental data of cavitating flows. This wealth of information can typically only be obtained from DNS or LES. TR-PIV offers insight into the dynamics of cavitating flows and will help facilitate the development of reliable and accurate numerical models based on actual cavity physics.

\section{Acknowledgments}

The authors gratefully acknowledge support by the National Science Foundation (NSF, Dr. M. Plesniak), the Office of Naval Research (ONR, Dr. Kam Ng). Thanks to TSI Inc. (Dr. I. Milosevic) for the use of their TR-PIV system. Thanks to D. Kawakami for help with experiments and E. Axdahl for help with image processing. Drs. Qin and Wosnik gratefully acknowledge support by the Minnesota Supercomputing Institute (MSI).

\section{References}

[1] Arndt REA (1981) Cavitation in fluid machinery and hydraulic structures. Ann Rev Fluid Mech 13:273-328

[2] Edstrand H (1950) "Cavitation Tests with Model Propellers in Natural Sea Water with Regard to Gas Content of Water and its Effect upon cavitation Point and Propeller Characteristics”, SSPPA Report No. 5, Göteborg, Sweden

[3] Kubota A, Kato H and Yamaguchi H (1992) A new modeling of cavitating flows: a numerical study of unsteady cavitation on a hydrofoil section, J Fluid Mech, 240:59-96.

[4] Song CCS, He J, Zhou F and Wang G (1997) Numerical simulation of cavitating and non-cavitating flows over a hydrofoil, SAFL project report, no.402, St. Anthony Falls Laboratory, University of Minnesota

[5] Song CCS and Qin Q (2001) Numerical Simulation of Unsteady Cavitating Flows, 4th International Symposium on Cavitation, Pasadena, California

[6] Qin Q, Song CCS and Arndt REA (2003) Numerical study of unsteady turbulent wake behind a cavitating hydrofoil, $5^{\text {th }}$ International Symposium on Cavitation, Osaka, Japan.

[7] Arndt REA, Song CCS and Qin Q (2004) Experimental and numerical investigations of cavitating hydrofoils. $22^{\text {nd }}$ IAHR Symposium on Hydraulic Machinery and Systems, Stockholm, Sweden, June 29-July 2, 2004.

[8] Qin Q (2004) Numerical Modeling of Natural and Ventilated Cavitating Flows, PhD Thesis, University of Minnesota. 
[9] Arndt REA, Song CCS, Kjeldsen M, He J, Keller A. (2000) Instability of partial cavitation; A numerical/experimental approach. In: Proc 23rd Symp on Naval Hydrodynamics. Washington DC: Academic Press

[10] de Lange GE de Bruin GH, and van Wijngaarden L (1994) On the mechanism of cloud cavitation - experiment and modeling. Proc. 2nd Intl. Symp. on Cavitation, Tokyo, Japan pp 45-50

[11] Kubota A, Kato H, Yamaguchi H, Maeda M (1989) Unsteady structure measurement of cloud cavitation on a foil section using conditional sampling technique. J Fluids Eng 111:204-210

[12] Arndt REA, Ellis C and Paul S (1995) Preliminary Investigation of the Use of Air Injection to Mitigate Cavitation Erosion. J Fluids Eng 117:498-592

[13] de Lange D (1996) Observation and Modeling of Cloud Formation Behind a Sheet Cavity. Ph.D. Dissertation, University

[14] Kawanami, Y., Kato, H., Yamaguchi, H., Tanimura, M.. and Tagaya, Y (1997) Mechanism and Control of Cloud Cavitation. J Fluids Eng, 119:788-794

[15] Watanabe S, Tsujimoto Y and Furukawa A (2001) Theoretical Analysis of Transitional and Partial Cavity Instabilities” J Fluids Eng 123:692-697

[16] Kjeldsen M, Arndt REA, Effertz M (2000) Spectral characteristics of sheet/cloud cavitation. J Fluids Eng 122:481487

[17] Laberteaux KR, Ceccio SL (2001a) Partial Cavity Flows. Part1. Cavities forming on models without spanwise variation. J Fluid Mech 431:1-41

[18] Laberteaux KR, Ceccio SL (2001b) Partial Cavity Flows. Part2. Cavities forming test objects with spanwise variation. J Fluid Mech 431:43-63

[19] Arndt REA, Kjeldsen M, Song CCS and Keller A (2002) Analysis of Cavitation Wake Flows, $21^{\text {st }}$ IAHR Symposium on Hydraulic Machinery and Systems, Lausanne, Switzerland, September 9-12, 2002.

[20] Arndt REA, Kawakami DT, Wosnik M (2006) Measurements in Cavitating Flows. In Handbook of Fluid Mechanics, eds. C. Tropea and J. Foss, Springer Verlag.

[21] Wosnik M, Schauer TJ, Arndt REA (2004) Experimental Investigation of the Turbulent Bubbly Wake in a Ventilated Flow, In: Advances in Turbulence X, H. I. (eds Andersson and P-A Krogstad) pp.657-660, Barcelona, CIMNE.

[22] Fontecha Gomez L (2004) PIV Measurements in the Wake of a Supercavitating Body, M.S. Thesis, Chalmers University of Technology, Gothenburg, Sweden (carried out at U of MN).

[23] Hart DP (1998) High-Speed PIV analysis using Compressed Image Correlation. J Fluids Eng, 120:463-470 